\title{
MERIDIAN \& ASTROLABE OBSERVATIONS OF RADIO STARS: A CONTRIBUTION TO THE CONNECTION OF RADIO AND OPTICAL REFERENCE SYSTEMS
}

\author{
M. Sarasso, ${ }^{1}$ G. Chiumiento, ${ }^{1}$ and A. Poma ${ }^{2}$ \\ 1 Osservatorio Astronomico di Torino \\ I-10025 Pino Torinese (TO), Italy \\ 2 Stazione Astronomica Internaz. di Latitudine \\ Via Ospedale 72 \\ I-09100 Cagliari, Italy
}

\begin{abstract}
Optical positions of eight radio stars observed with the Photoelectric Transit Instrument (PTI) at Torino and with the Danjon Astrolabe (AST) at Cagliari are presented here. The values of observed right ascensions have been computed at the Epoch J2000.0.
\end{abstract}

Precise positions of optical counterparts of radio sources are needed for the connection of optical and radio reference frames.

The optical counterparts of eight galactic radio sources-from the list proposed by the IAU Commission 24 Working Group on the identification of RadioOptical Astrometric Sources-have been regularly observed with the Photoelectric Transit Instrument at Torino Observatory. The radio source HR 5110 has also been observed, at west transit only, with the Danjon Astrolabe at Cagliari Observatory.

The observations, obtained in different periods, have been reduced according to the MERIT Standards, that is, on the basis of the IAU (1976) System of Astronomical Constants, the IAU (1980) Theory of Nutation, and the FK5 System.

The optical positions of seven of the above galactic radio stars - at the epoch J2000.0 - are presented in Table 1 as well as a comparison with right ascensions obtained with Carlsberg Meridian Circle at La Palma (1986).

In particular, with reference to the radio source HR 5110, the values of right ascensions, computed at Torino and Cagliari at the epoch $\mathrm{J} 2000.0$, are presented in Table 2 as well as a comparison with optical positions obtained with the observations for the Astrolabe General Catalogue (Billaud et al., 1977), with Transit Instrument (TI) at Bratislava (Hefty et al., 1987) and the Automatic Meridian Circle (AMC) at Bordeaux Observatory (Lestrade et al., 1985).

Finally, a preliminary comparison with VLBI radio position of this source, measured with the NASA-JPL Deep Space Network and the US VLB Network antennas, is also presented. 
Table 1: OPTICAL POSITIONS OF GALACTIC RADIO SOURCES OBSERVED AT TORINO AND LA PALMA OBSERVATORIES

\begin{tabular}{|c|c|c|c|c|c|c|c|c|c|c|c|}
\hline Source & DH & - & sp & $\alpha(0-c)$ & & Qobs & $\alpha_{\text {calc }}$ & $\delta$ calc & N & Ep. & Cat \\
\hline b Per & $49^{\circ} 1150$ & 4.56 & $A 2$ & $\begin{array}{l}-0.0379 \\
\pm 0.0049\end{array}$ & $h^{h} 18^{m}$ & $\begin{array}{l}m_{14.604} \\
14.522\end{array}$ & 14.642 & $50^{\circ} 17^{\prime} 44^{\prime \prime} .05$ & $\begin{array}{r}20 \\
7\end{array}$ & $\begin{array}{l}84.37 \\
85.11\end{array}$ & $\begin{array}{l}\text { (1) } \\
\text { (2) }\end{array}$ \\
\hline $54 \mathrm{Can}$ & 571118 & 6.49 & 60 & $\begin{array}{l}-0.0193 \\
\pm 0.0044\end{array}$ & 82 & $\begin{array}{l}35.809 \\
35.842\end{array}$ & 35.828 & $5716 \quad 24.67$ & $\begin{array}{r}22 \\
6\end{array}$ & $\begin{array}{l}83.79 \\
85.73\end{array}$ & $\begin{array}{l}\text { (1) } \\
\text { (2) }\end{array}$ \\
\hline $22 \mathrm{BOO}$ & 262508 & 6.80 & FO & $\begin{array}{r}0.1076 \\
\pm 0.0055\end{array}$ & 1356 & $\begin{array}{l}9.505 \\
9.622\end{array}$ & 9.397 & $2555 \quad 7.41$ & $\begin{array}{l}6 \\
7\end{array}$ & $\begin{array}{l}82.46 \\
85.04\end{array}$ & $\begin{array}{l}\text { (1) } \\
\text { (2) }\end{array}$ \\
\hline$\beta$ Lyr & 333223 & $3.4-4.3$ & $B p$ & $\begin{array}{r}0.0001 \\
\pm 0.0059\end{array}$ & 1850 & 4.802 & 4.802 & 332145.71 & 4 & 81.74 & FK4 \\
\hline P Cyg & 373871 & 4.77 & $B p$ & $\begin{array}{r}0.0479 \\
\pm 0.0038\end{array}$ & 2017 & 47.219 & $\begin{array}{l}47.171 \\
47.211\end{array}$ & $38 \quad 158.85$ & $\begin{array}{r}21 \\
6\end{array}$ & $\begin{array}{l}83.73 \\
85.69\end{array}$ & SAO \\
\hline HD 216489 & 164831 & 5.84 & KO & $\begin{array}{l}-0.0056 \\
\pm 0.0035\end{array}$ & 2253 & 2.291 & $\begin{array}{l}2.297 \\
2.292\end{array}$ & 165027.86 & $\begin{array}{r}37 \\
6\end{array}$ & $\begin{array}{l}83.76 \\
85.52\end{array}$ & $\begin{aligned} & \text { FK4S (1) } \\
& \text { (2) }\end{aligned}$ \\
\hline HR 8752 & 562923 & 4.96 & GO & $\begin{array}{r}0.0691 \\
\pm 0.0101\end{array}$ & 2300 & 5.102 & $\begin{array}{l}5.033 \\
5.112\end{array}$ & $56 \quad 56 \quad 43.12$ & $\begin{array}{l}4 \\
6\end{array}$ & $\begin{array}{l}82.07 \\
85.55\end{array}$ & $\begin{aligned} \text { FK4S (1) } & \text { (2) }\end{aligned}$ \\
\hline
\end{tabular}

(1) Photoelectric Transit Instrunent - Torino Observatory - (2) Carlsberg Meridian Circle - La Palea $N=$ Nunber of observ. $\quad E p=$ Hean epoch of observ. $(1980+)$ Cat = Optical Ref. adopled Catalogue

Table 2: OPTICAL AND RADIO POSITIONS OF RADIO SOURCE HR $5110=$ DH $372426=$ FK4 $502 \quad n=4.96 \quad 5 p$. FO

\begin{tabular}{|c|c|c|c|c|c|c|c|c|}
\hline Station & $\alpha(0-c)$ & $\varepsilon$ & Qobs & $\alpha$ calc & $\delta_{\text {obs }}$ & $\delta$ calc & $N$ & Ep. \\
\hline Torino PII & $0.5009+1$ & -0.0020 & $13^{\mathrm{h}} 34_{4}^{\mathrm{m}} \mathrm{S}_{\mathrm{s}} .803$ & $4 T .802$ & & $37^{\circ} 10^{\prime} 56^{\prime \prime} .70$ & 19 & 82.67 \\
\hline Cagliari AST & 0.0105 & 0.0036 & 47.812 & 47.802 & & 56.70 & 21 & 84.09 \\
\hline G.C.A. & 0.0021 & 0.0007 & 47.804 & 47.802 & & & & 64.50 \\
\hline Bratislava TI & 0.0004 & 0.0035 & 47.802 & 47.802 & & & & 76.20 \\
\hline Bordeaux AnC & 0.0090 & & 47.695 & 47.686 & $37^{\circ} 10^{\prime} 56^{\prime \prime} .92$ & 56.92 & & 83.32 \\
\hline VLBI Netvork & & & 47.689 & & 56.86 & & & 83.57 \\
\hline
\end{tabular}

* To conpute this positions, proper otions and trigonocetric parallaxes have not been taken into account, between the epoch of observation and J2000.0

References

1) BILLAUD et Al.: 1977, Astron. Astrophys. 63, 87

2) LESTRADE et Al.: 1985, Proc. Coll. Scientific Aspects of the Input Catalogue preparation'. Aussois, 3-7 June 1985,251 (ESA SP-234)

3) CARLSBERG MERIDIAN CATALOGUE - La Palma, No. 2, 1986

4) HEFTY et Al.: 1987, Bull. Astr. Inst. Czechosl. 38, 24 